\title{
Mitigation of Physical Aging with Mixed Matrix Membranes Based on Cross-Linked PIM-1 Fillers and PIM-1
}

\author{
Marzieh Tamaddondar, Andrew B. Foster, Mariolino Carta, Patricia Gorgojo, Neil B. McKeown,
} and Peter M. Budd*

Cite This: ACS Appl. Mater. Interfaces 2020, 12, 46756-46766

Read Online

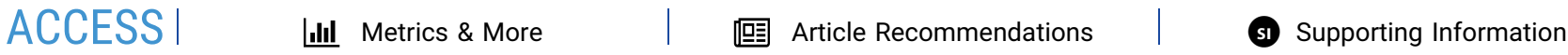

ABSTRACT: A low cross-link density (LCD) network-PIM-1, which offers high compatibility with the polymer of intrinsic microporosity PIM-1, is synthesized by a modified PIM-1 polycondensation that combines both a tetrafluoro- and an octafluoro-monomer. To maximize the advantages of utilizing such cross-linked PIM-1 fillers in PIM-1-based mixed matrix membranes (MMMs), a grafting route is used to decorate the LCD-network-PIM-1 (dispersed phase) with PIM-1 chains, to further enhance compatibility with the PIM-1 matrix. Mixed-gas $\mathrm{CO}_{2} / \mathrm{CH}_{4}(1: 1, \mathrm{v} / \mathrm{v})$ separation results over 160 days of membrane aging confirm the success of a relatively short $(24 \mathrm{~h})$ grafting reaction in improving the initial $\mathrm{CO}_{2}$ separation

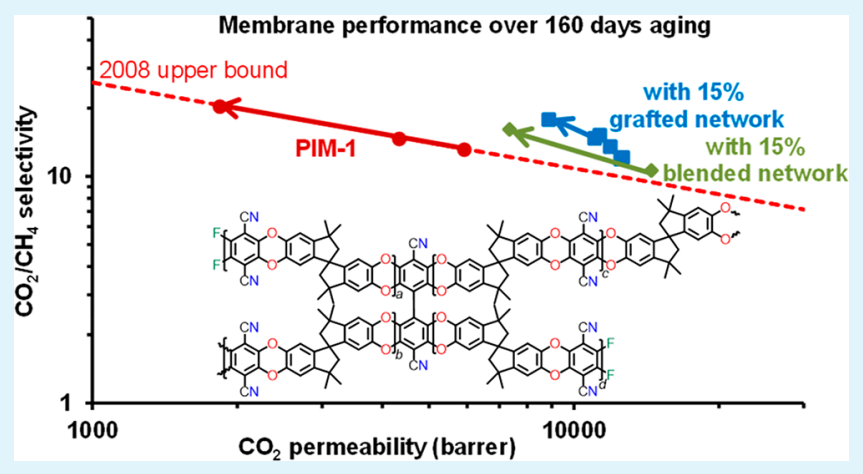
performance, as well as hindering the aging of PIM-1/graftedLCD-network-PIM-1 MMMs. For MMMs based on a $24 \mathrm{~h}$ grafting route, all the gas separation data surpass the 2008 Robeson upper bound by a significant margin, and the 160-day aged membranes show only $29 \%$ reduction from the initial $\mathrm{CO}_{2}$ permeability, which is substantially less than the equivalent losses of nearly $70 \%$ and $48 \%$ for PIM-1 and traditionally fabricated MMMs counterparts, respectively. These results demonstrate the potential of network-PIM components for obtaining much more stable gas separation performance over extended periods of time.

KEYWORDS: polymer of intrinsic microporosity (PIM), mixed matrix membrane, grafting, cross-linking, membrane aging, $\mathrm{CO}_{2}$ separation

\section{INTRODUCTION}

In today's carbon-constrained world, membrane-based gas separation has a great deal to offer when compared to conventional energy-intensive separation processes such as pressure swing adsorption and distillation. ${ }^{1,2}$ High free volume, glassy polymers such as polymers of intrinsic microporosity (PIMs), and specifically the archetypal member of the family, PIM-1, have shown a promising combination of permeability coefficient and permselectivity. ${ }^{3}$ The rigid polymeric chains of PIM-1, with spiro-center sites of contortion, are unable to pack efficiently, leaving a large amount of interconnected free volume that gives rise to microporosity (pore size $<2 \mathrm{~nm}$, as defined by IUPAC). ${ }^{4}$ Analogous to molecular sieves, this class of rigid, ladder-type polymers with irregularly shaped micropores overcame the 1991 Robeson trade-off relationship ${ }^{5}$ between selectivity and permeability for key gas pairs, and contributed to a revision of the upper bounds in $2008 .^{6}$

Following on from Freeman's theoretical prediction of strategies in material design for surpassing the upper bound, further optimization of polymer structure has improved the balance between permeability and selectivity by tuning the interchain rigidity and interchain spacing in the gas-sieving ultramicroporous region of PIM-like polymers. Through careful design of the polymeric backbone and incorporation of groups such as triptycene (Trip), ethanoanthracene (EA), Tröger's base (TB), and tetramethyltetrahydronaphthalene (TMN),$^{8-10}$ new upper bounds were introduced in 2015 for some gas pairs, ${ }^{11}$ and recently in 2019 for $\mathrm{CO}_{2} / \mathrm{CH}_{4}$ and $\mathrm{CO}_{2} /$ $\mathrm{N}_{2}$ mixtures. $^{12}$

Very recently in our group, a comprehensive study was conducted to help understand the importance of the topology of PIM-1 structures in tuning the membrane performance. ${ }^{13}$ On the basis of PIM-1 synthesis utilizing tetrachloroterephthalonitrile (TCTPN), instead of costlier tetrafluoroterephthalonitrile (TFTPN), in a step-growth polymerization with 5,5',6,6'-tetrahydroxy-3,3,3',3'-tetramethyl-1,1' -spirobisindane (TTSBI), significantly different PIM-1 structures (including

Received: August 1, 2020

Accepted: September 9, 2020

Published: September 9, 2020

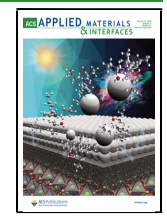


(a)

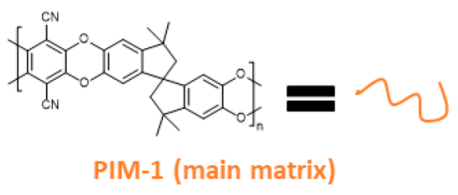

(c)

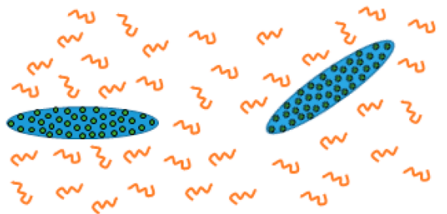

(b)

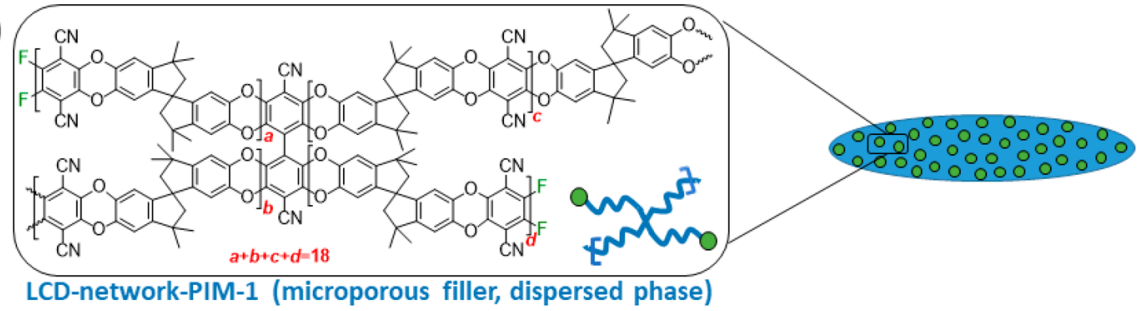

Possible reaction sites (fluorine end groups) of LCD-network-PIM-1 with TTSBI monomer

Wrafted PIM-1 chain
Mixture of PIM-1 and LCD-network-PIM-1 for casting into MMMs with possibility of chain relaxation over time

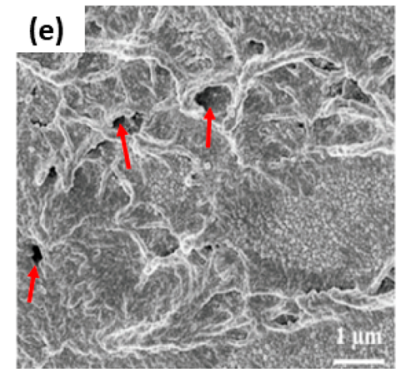

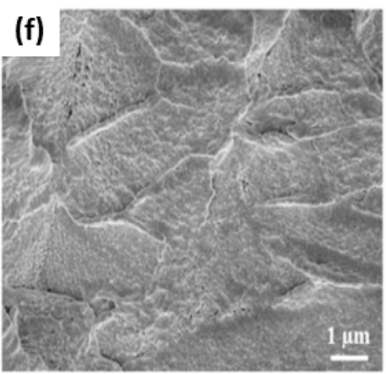

(d)

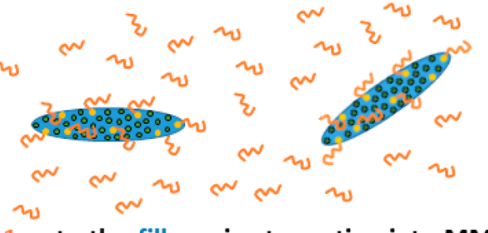

Grafting PIM-1 onto the filler prior to casting into MMMs for potential restriction of PIM-1 chain movements over time

Figure 1. (a) Chemical structure and simplified representation of PIM-1, (b) proposed chemical structure and simplified representation of low cross-link density (LCD) network-PIM-1 which contains fluorine end groups that may act as sites for further reaction (shown by green dots in representation), (c) schematic representation of blend mixture of PIM-1 and LCD-network-PIM-1 used for preparation of MMMs, (d) schematic representation of PIM-1-grafted onto LCD-network-PIM-1 which was used for preparation of MMMs which could be compared against the simple blend mixture shown in (c); and cross sectional SEM images of (e) a conventionally prepared MMM based on blending PIM-1 and 15 wt \% LCDnetwork-PIM-1 (BMM-15\%), with red arrows showing possible defects formed between the two phases, and (f) and (g) MMMs prepared via polymerization of PIM-1 in the presence of LCD-network-PIM-1 over $24 \mathrm{~h}$ (PMM-24h) and $72 \mathrm{~h}$ (PMM-72h), respectively.

cyclic, branched, and network topologies) were prepared, and a positive correlation was observed between the colloidal network content of PIM-1 and the $\mathrm{CO}_{2} / \mathrm{N}_{2}$ selectivity. Of the PIM-1 types prepared in that work, the one with the highest network content delivered a performance which even surpassed the recently proposed 2019 upper bound for $\mathrm{CO}_{2} /$ $\mathrm{CH}_{4}$ separation.

The abundant pore volume of PIM-1, although highly beneficial in delivering initial high gas separation performance, can also be detrimental to the permeability coefficient over extended periods of time. Relaxation of the polymeric chains of glassy polymers such as PIM-1 over time from a nonequilibrium state to a more energetically favored state, referred to as "physical aging", reduces the fractional free volume of the polymer and is manifested by a decrease in gas permeability and often an increase in selectivity. Such instability in the longterm separation performance of PIM-1-based membranes has been the hurdle in their commercialization. Consequently, a large body of research has sought ways to control or mitigate aging by preserving the initial high fractional free volume of highly microporous polymers such as PIM-1 and poly(1trimethylsilyl-1-propyne) (PTMSP). ${ }^{14}$

Rigidification of the polymer structure by various chemical and thermal cross-linking routes ${ }^{15-18}$ has been explored for PIM-1-based membranes with the hope of retarding aging. Although cross-linking the polymeric chains has proven, to some extent, successful in inhibiting chain relaxation and reduction of fractional free volume, ${ }^{17,19}$ it can also come at the cost of sacrificing the initial high permeability. ${ }^{18}$
Introduction of additives such as nanoparticles and microporous organic fillers has also been among the promising approaches for inhibition of aging. Mixed matrix membranes (MMMs) based on PIM-1 and various organic and inorganic fillers have been studied for gas separation. ${ }^{20-24}$ Inclusion of certain fillers, such as functionalized graphene and graphene oxide (GO), ${ }^{25}$ hyper-cross-linked polymer (HCP), ${ }^{26-28}$ and polyaromatic framework (PAF-1 $)^{29}$ has shown promising effects toward impeding or even ending aging. It has been shown that the similar composition of nanoporous organic fillers, or more specifically the aryl-H groups of PAF-1 and methyl groups of PIM-1 and PTMSP, result in formation of molecular interactions at the interface and therefore an antiaging effect in the MMMs. ${ }^{30}$

In view of the advantages of porous organic materials, the key to the evolution of PIM-1-based MMMs may be the design of new organic fillers that not only facilitate intimate mixing between the filler and the main PIM-1 matrix to reduce interfacial defects, but also freeze the micropores in place and minimize membrane aging. MMMs containing Metal Organic Frameworks (MOFs) and functionalized MOFs have shown promising enhancement of the gas separation performance and in particular $\mathrm{CO}_{2} / \mathrm{CH}_{4}$ adsorption selectivity. ${ }^{31,32}$ Fabrication of the majority of MMMs is based on simple "mixing and casting" routes which may contribute to the formation of defects and unselective voids at the interface of the two phases in the final membrane. This has been addressed in recent work by Tien-Binh and co-workers ${ }^{33}$ in which the authors proposed an alternative route for fabrication of PIM-1-based MMMs. 
Upon addition of UiO-66- $\mathrm{NH}_{2}$ particles to PIM-1 reaction media, MOF particles were bonded to the growing PIM-1 chains by a favorable chemical linkage between the amine group of UiO-66- $\mathrm{NH}_{2}$ and the TFTPN monomer used for PIM-1 synthesis. The authors termed the route as an "in situ cross-interface linking" between PIM-1 and MOF particles and showed that the improved interfacial compatibility of the two phases of the as-prepared MMMs results in higher solubility and diffusivity selectivity and a molecular sieving behavior in gas separation. More importantly, the report includes $\mathrm{CO}_{2} /$ $\mathrm{CH}_{4}$ gas separation data for 400-day aged MMMs which demonstrates considerably controlled aging (20\% loss of the initial $\mathrm{CO}_{2}$ permeability compared to up to $80 \%$ loss for PIM-1 counterparts) due to the restriction of free movements of PIM1 chains over time.

In the pursuit of a porous organic filler which could potentially offer maximum compatibility with PIM-1, we recently introduced a tightly cross-linked version of PIM-1 (network-PIM-1) ${ }^{34}$ for application in PIM-1-based MMMs. This utilized the effectively tetrafunctional monomer dicyanooctafluoro biphenyl (DCOB), instead of the effectively bifunctional monomer TFTPN, in a step-growth polymerization with TTSBI. To our surprise, a variety of characterization methods elucidated that network-PIM-1 was composed of nanosheet structures. These did not disperse well in the PIM-1 matrix at high loading, but produced a noticeable improvement in $\mathrm{CO}_{2} / \mathrm{CH}_{4}$ separation performance when used at very low loading. This was analogous to the gas separation performance of some other PIM-1-based MMMs containing low loadings of high aspect ratio fillers such as graphene and graphitic carbon nitride nanosheets. ${ }^{35,36}$

Building upon our previous research, here we describe a modified version of network-PIM-1 with considerably lower cross-link density than the previously reported network structure, synthesized using a combination of TFTPN and DCOB with TTSBI. This is referred to as LCD-network-PIM1. We also sought to effectively graft additional PIM-1 chains onto this microporous filler, by including the filler, which has unreacted fluoro- end groups, in a PIM-1 synthesis with TFTPN and TTSBI. Two reaction times were investigated, 24 and $72 \mathrm{~h}$, yielding the reaction products, denoted as polymerized mixed matrix (PMM) of PMM-24h and PMM$72 \mathrm{~h}$, respectively. The polymerized PIM-1/filler mixtures were used for fabrication of composite membranes. Our hypothesis was that grafting would enhance compatibility between the network and the PIM-1 matrix, minimizing the formation of defects and voids at the interface and constraining mobility of the PIM-1. For comparison, a simple blending and casting route was also used to prepare MMMs of ungrafted LCDnetwork-PIM-1 with PIM-1. The blended MMMs are denoted as $\mathrm{BMM}-\mathrm{X} \%$, where $X$ stands for the weight percentage LCDnetwork-PIM-1 of the total solids content.

The two approaches, blending and grafting, are summarized in Figure 1 $(a-d)$. We show here that composite membranes based on PIM-1 grafted to LCD-network-PIM-1 in a $24 \mathrm{~h}$ reaction demonstrate particularly good $\mathrm{CO}_{2} / \mathrm{CH}_{4}$ separation performance, combined with noticeably controlled aging over 160 days. These results pave the way toward production of PIM-1-based membranes which can offer long-term stable performance for industrial applications.

\section{EXPERIMENTAL SECTION}

2.1. Synthesis of Network-PIM-1 with Low Cross-Link Density (LCD-Network-PIM-1). Network-PIM-1 with low crosslink density, denoted as LCD-network-PIM-1, was synthesized by the polycondensation reaction of a mixture of tetrafluoroterephthalonitrile (TFTPN) and dicyanooctafluoro biphenyl (DCOB) with 5,5',6,6'tetrahydroxy-3,3,3',3'-tetramethyl-1,1'-spirobisindane (TTSBI). The synthesis procedure for $\mathrm{DCOB}$ can be found in the Supporting Information (SI). Considering that DCOB has twice as many functional groups as TFTPN, the starting molar ratio of TFTPN/ $\mathrm{DCOB}$ was set to $0.95 / 0.025$. If the reaction proceeds to full conversion and a complete network structure forms, then it will be composed on average of a total of 40 TTSBI residues and 38 TFTPN residues connected around every fully reacted DCOB unit (see Figure 2).

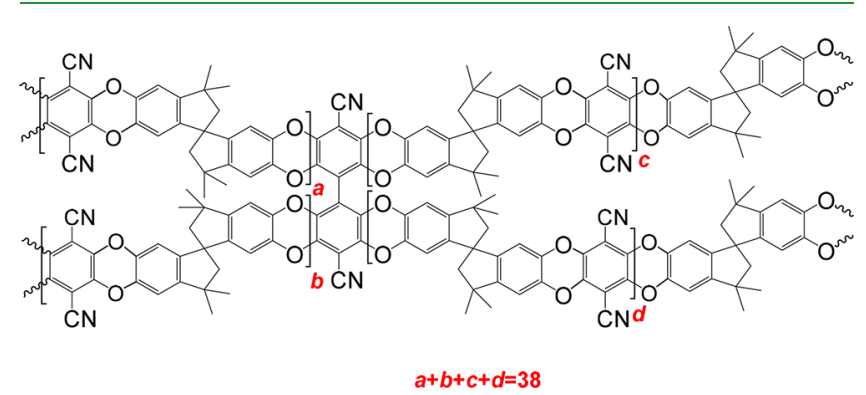

Figure 2. An estimation of the theoretical repeat unit of LCDnetwork-PIM-1 based on the starting composition of TFTPN/ DCOB: $0.95 / 0.025$.

The detailed synthesis route for preparation of the LCD-networkPIM-1 is as follows:

LCD-network-PIM-1 was synthesized using purified and well-dried monomers and potassium carbonate. To a $100 \mathrm{~mL}$ round-bottom flask equipped with a Dean-Stark trap, TFTPN (1.088 g, 5.44 $\mathrm{mmol})$, DCOB (0.0498 g, $0.143 \mathrm{mmol})$, TTSBI (1.95 g, $5.73 \mathrm{mmol})$, and potassium carbonate $\left(\mathrm{K}_{2} \mathrm{CO}_{3}, 3.166 \mathrm{~g}, 22.91 \mathrm{mmol}\right)$ were added, and the mixture was stirred under dry $\mathrm{N}_{2}$ at room temperature for 30 min. The mixture was kept stirring at room temperature while anhydrous dimethylacetamide (DMAc, $25 \mathrm{~mL}$ ) and dichlorobenzene (DCB, $13 \mathrm{~mL}$ ) were added to the mixture. A bath was then set to 160 ${ }^{\circ} \mathrm{C}$, and the round-bottom flask containing the reaction mixture was mounted in it when a temperature of $120{ }^{\circ} \mathrm{C}$ was reached. The reaction was continued for a total of $40 \mathrm{~min}$, during which the mixture turned highly viscous and further amounts of DMAc $(10 \mathrm{~mL})$ and DCB $(5 \mathrm{~mL})$ were added to the reaction mixture to avoid the premature termination of the reaction. The mixture was then quenched in deionized (DI) water and 2-3 drops of very dilute $\mathrm{HCl}$, after which the precipitate was isolated and dried under reduced vacuum ( $3 \mathrm{~h}$ at room temperature). The crude polymer was washed with acetone $(200 \mathrm{~mL})$ and then methanol $(300 \mathrm{~mL})$. After the filtered product was well dried under vacuum at room temperature, the polymer was further purified using a reflux condenser overnight with different solvents in a sequence of dimethylformamide (DMF, $400 \mathrm{~mL}$ ), tetrahydrofuran (THF, $400 \mathrm{~mL})$, chloroform $\left(\mathrm{CHCl}_{3}, 400\right.$ $\mathrm{mL}$ ), acetone $(400 \mathrm{~mL})$, and two times methanol $(400 \mathrm{~mL})$. The product was filtered while still hot after each reflux with a fresh solvent and was dried thoroughly under suction at room temperature for $2 \mathrm{~h}$. Before being refluxed again, the polymer was washed with the next solvent. Finally, the polymer was dried overnight in a vacuum oven at $110{ }^{\circ} \mathrm{C}$ to give $2.1907 \mathrm{~g}$ (83\%) of LCD-network-PIM-1, elemental analysis results: C: $73.35 \%, \mathrm{H}: 4.42 \%, \mathrm{~N}: 5.87 \%$. The elemental ratios suggest 19 TTSBI, 18 TFTPN for every fully reacted DCOB monomer (further details can be found in SI Table S1). This means that the final recovered product has a slightly higher cross-link density than expected based on the starting monomer mixture. A possible theoretical repeat unit of LCD-network-PIM-1 based on the elemental 
analysis is presented in Figure 3. It can be inferred that the network structure has some unreacted fluoro- end groups.

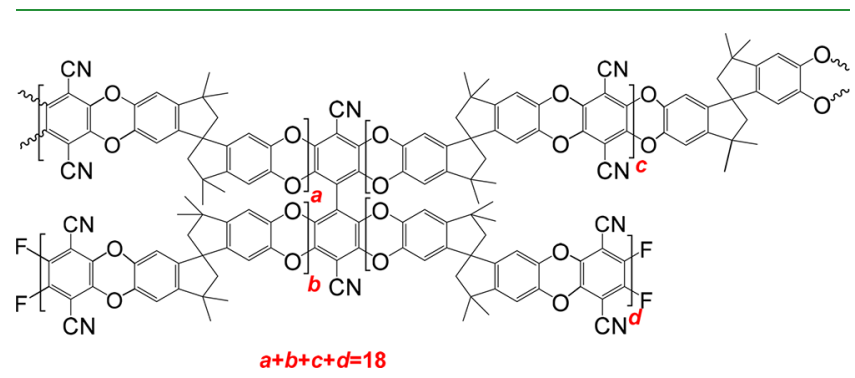

Figure 3. An estimation of the actual repeat unit of the LCD-networkPIM-1 determined from the elemental analysis results of the product.

2.2. Grafting Reaction of LCD-Network-PIM-1 and PIM-1. Two polymerizations to give PIM-1 were carried out in the presence of LCD-network-PIM-1. The initial hypothesis for this was that the potentially available fluoro- end groups of LCD-network-PIM-1 can readily react with the TTSBI monomer used in PIM-1 synthesis, therefore facilitating grafting of PIM-1 chains onto LCD-networkPIM-1. The experimental details of the grafting reaction are as follows:

TFTPN, TTSBI, $\mathrm{K}_{2} \mathrm{CO}_{3}$, and LCD-network-PIM-1 were all well dried in a vacuum oven at room temperature over 3 days. TFTPN $(0.4090 \mathrm{~g}, 2 \mathrm{mmol})$, TTSBI $(0.6959 \mathrm{~g}, 2 \mathrm{mmol})$, LCD-network-PIM$1(0.158 \mathrm{~g})$, and $\mathrm{K}_{2} \mathrm{CO}_{3}(0.8475 \mathrm{~g}, 6 \mathrm{mmol})$ were added to a threeneck round-bottom flask, and the mixture was stirred under dry $\mathrm{N}_{2}$ at room temperature for $1 \mathrm{~h}$. Following this, $15 \mathrm{~mL}$ of anhydrous DMF was added to the mixture, and the temperature was set at $70{ }^{\circ} \mathrm{C}$. Similar reactions were carried out for total reaction times of 24 and 72 h. At the end of each reaction, the mixture was poured into methanol, followed by filtering and drying the precipitate under reduced pressure for a total of $3 \mathrm{~h}$ at room temperature. To remove the remaining salts and any low molar mass oligomers, the reaction product was further purified by overnight reflux in DI water, followed by three times washing and filtration with acetone and methanol. Finally, the reaction products were dried in a vacuum oven at $110^{\circ} \mathrm{C}$ for $15 \mathrm{~h}$ to obtain final yields of 1.0085 and $0.9698 \mathrm{~g}$ after 24 or $72 \mathrm{~h}$ reaction time, respectively. The resulting polymers were denoted as PMM-24h and PMM-72h, to reflect their respective grafting reaction times of 24 and $72 \mathrm{~h}$, respectively. Elemental analysis results for PMM-24h: C: $75.25 \%, \mathrm{H}: 4.68 \%, \mathrm{~N}: 5.76 \%$, and for PMM-72h: C: $72.47 \%, \mathrm{H}: 4.12 \%, \mathrm{~N}: 5.90 \%$. Assuming that the loss of the crosslinked filler particles (LCD-network-PIM-1) during the purification steps is negligible, a calculation suggests that both final products, i.e., PMM-24h and PMM-72h, contain nearly 15 wt \% LCD-networkPIM-1 in the whole final solid product.

2.3. Membrane Fabrication. PIM- $1\left(M_{\mathrm{w}}=158 \mathrm{~kg} \mathrm{~mol}^{-1}, \boxplus=\right.$ 2.95 , synthesis details are provided in the SI) was used for the fabrication of control PIM-1 membranes (0.3 g PIM- 1 in $10 \mathrm{~mL}$ chloroform) as well as the conventional free-standing blend MMMs. For the conventional blending route, PIM-1 solution and LCDnetwork-PIM-1 dispersions were separately prepared in chloroform, which were then mixed and cast into films. Two MMMs with 15 and $30 \mathrm{wt} \%$ LCD-network-PIM-1 in the total solid content were prepared via the blending approach. In the second method, however, the purified products of the 24-h and 72-h grafting reactions, each containing nearly $15 \mathrm{wt} \%$ LCD-network-PIM-1 in the total solid content, were directly cast into membranes without any further addition of LCD-network-PIM-1 or PIM-1.

As an example, detailed procedures related to the preparation of MMMs containing $15 \mathrm{wt} \%$ of the LCD-network-PIM-1 based on each method is presented here:

For the blend MMMs, PIM-1 (0.3 g) was dissolved in anhydrous chloroform $(5 \mathrm{~mL})$ through magnetically stirring the solution at 300 rpm and room temperature for one night. In a separate vial, $0.0529 \mathrm{~g}$ of LCD-network-PIM-1 was dispersed in $5 \mathrm{~mL}$ anhydrous chloroform by overnight stirring ( $300 \mathrm{rpm})$, followed by $10 \mathrm{~min}$ sonication using a probe sonicator (Cole-Parmer Instruments, CPX 750, $750 \mathrm{~W}$ ). The two samples were then mixed by magnetic stirring for $12 \mathrm{~h}$ followed by sonication for $10 \mathrm{~min}$. The dispersion was then cast in a glass Petri dish (diameter: $7 \mathrm{~cm}$ ) and was kept in a closed compartment under a controlled flow of dried nitrogen for slow evaporation of the solvent over 3 days. The dried films, containing 15 wt \% LCD-network-PIM1 , were then soaked in methanol for a total of $18 \mathrm{~h}$, after which they were dried at room temperature for $10 \mathrm{~h}$ and then under vacuum at $110{ }^{\circ} \mathrm{C}$ for $12 \mathrm{~h}$. The resulting film was designated as BMM- $15 \%$, where 15 stands for the weight percentage of LCD-network-PIM-1 with regard to the total solids content.

In the second method, $0.3 \mathrm{~g}$ of PMM-24h or PMM-72h, obtained from the grafting reactions of PIM-1 and LCD-network-PIM-1 for either 24 or $72 \mathrm{~h}$, was dispersed in $10 \mathrm{~mL}$ anhydrous chloroform. The colloidal mixture was then stirred for one night at $300 \mathrm{rpm}$ at room temperature, followed by $10 \mathrm{~min}$ sonication using a sonication probe (Cole-Parmer Instruments, CPX 750, $750 \mathrm{~W}$ ). The procedure for casting the dispersion, methanol treating and drying the films was exactly the same as for the BMMs. The MMMs prepared by this method were denoted by the same name as the reaction products, PMM-24h or PMM-72h, as each reaction product can be considered to be essentially a mixed matrix. Photographs of the as-cast PMM-24h and BMM-15\% membranes can be viewed in Figure S12.

2.4. Characterization Methods. Multidetector size exclusion chromatography (SEC) was undertaken using a Viscotek GPCmax VE2001 solvent/sample module with TDA302 triple detector array, with two Polymer Lab mixed bead columns (PL Mixed B $\times 2$ ). Samples were prepared in chloroform $\left(1 \mathrm{mg} \mathrm{mL}^{-1}\right)$, left to dissolve for $24 \mathrm{~h}$, and then passed through a PTFE syringe filter $(0.45 \mu \mathrm{m}$ pore diameter) prior to SEC analysis.

${ }^{1} \mathrm{H}$ nuclear magnetic resonance (NMR) spectra were collected with a Bruker DPX $400 \mathrm{MHz}$ spectrometer at room temperature.

Fourier transform infrared (FTIR) spectra of the polymers and monomers were collected using a Thermoscientific Nicolet iS5, iD5 spectrometer annexed to a Whatman FTIR purge gas generator. The spectra were recorded in the attenuated total reflectance (ATR) mode, with a resolution of $0.25 \mathrm{~cm}^{-1}$, a sensitivity of 1 , and 16 scans in the range $4000-400 \mathrm{~cm}^{-1}$.

Tapping mode atomic force microscopy (AFM) was carried out with a Bruker Multimode8 instrument. A dilute dispersion of the LCD-network-PIM-1 in chloroform was prepared via magnetic stirring for nearly $10 \mathrm{~h}$ followed by a short sonication of nearly 5 min using a Cole-Parmer Instruments CPX 750. A drop of the dispersion was cast on a piece of clean silicon wafer, which was washed by 15 min sonication in acetone, DI water, and propan-2-ol in advance. The coated wafer was then mounted on a $15 \mathrm{~mm}$ AFM sample mounting disk (Bruker, model SD-102) for the analysis. 2D surface scanning images were computed automatically by Nanoscope Analysis software. Gwyddion 2.50 software was used for the measurement of particle size and thickness.

A Micrometrics ASAP 2020 sorption analyzer was used to obtain $\mathrm{N}_{2}$ adsorption/desorption isotherms at $77 \mathrm{~K}$ and to measure the BET surface areas, using approximately $0.10 \mathrm{~g}$ of well-dried samples. Samples were initially degassed at $120^{\circ} \mathrm{C}$ for $16 \mathrm{~h}$ under high vacuum $\left(10^{-5} \mathrm{bar}\right)$ before starting the analysis. Samples were then reweighed and placed in the analysis port.

A PerkinElmer thermogravimetric analysis (TGA) System was used for the analysis of thermal stability of the synthesized polymers, using a heating rate of $10{ }^{\circ} \mathrm{C} \mathrm{min}{ }^{-1}$ under nitrogen atmosphere.

Particle size distribution of the polymers (50 ppm dispersions in chloroform prepared via magnetic stirring for nearly $10 \mathrm{~h}$ and a short sonication of nearly $5 \mathrm{~min}$ ) was measured by Dynamic Light Scattering (DLS) using a Malvern Zetasizer Nano ZS instrument. Measurements were done by setting the standard operating procedure (SOP) using polystyrene latex as the material for calibration. The dispersant was chosen as chloroform at $20^{\circ} \mathrm{C}$ and the standard glass cuvette (PCS1115) was used for the measurements.

$\mathrm{C}, \mathrm{H}$, and $\mathrm{N}$ elemental analysis was carried out using a Thermo Fisher Scientific Flash 2000 Organic Elemental Analyzer. Samples 

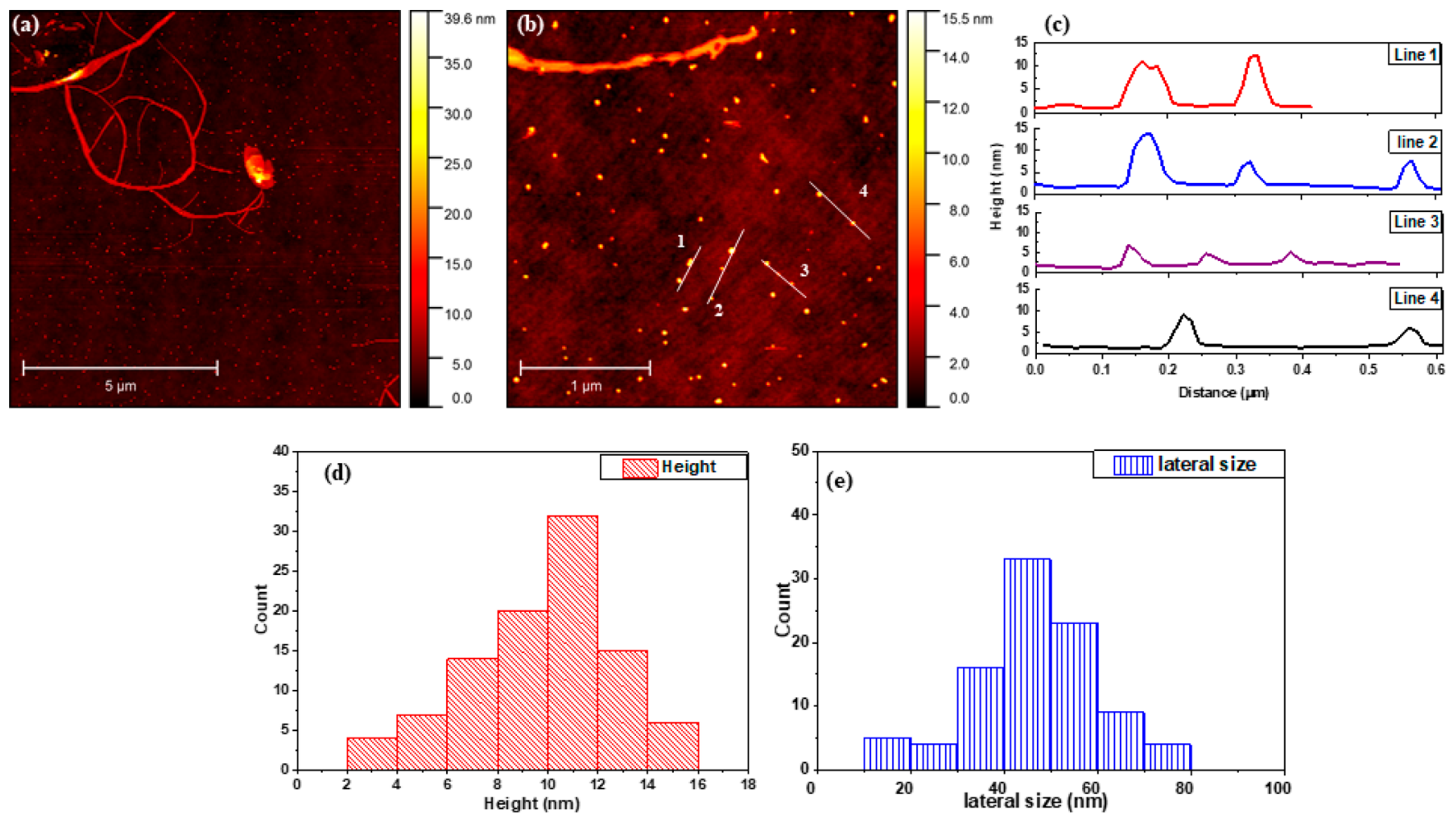

Figure 4. Analysis of LCD-network-PIM-1 particles; ( $a$ and b) AFM images at two different resolutions, (c) the height profiles related to lines 1 to 4 in image (b), and ( $\mathrm{d}$ and e) statistical analysis results for the height and the lateral size of LCD network PIM-1 particles, respectively.

Table 1. $\mathrm{CO}_{2} / \mathrm{CH}_{4}$ Mixed-Gas $(1: 1, v / v)$ Separation Results Obtained for PMM-24h, PMM-72h, BMM-15\%, BMM-30\%, and Pure PIM-1 Membranes ${ }^{a}$

\begin{tabular}{|c|c|c|c|c|}
\hline membrane & thickness $(\mu \mathrm{m})$ & filler content with respect to total solids (wt \%) & $\mathrm{CO}_{2}$ permeability (barrer) & $\mathrm{CO}_{2} / \mathrm{CH}_{4}$ selectivity \\
\hline PIM-1 & $59 \pm 10$ & 0 & $5920 \pm 380$ & $13.1 \pm 0.4$ \\
\hline PMM-24h & $140 \pm 16$ & 15 & $12510 \pm 1970$ & $11.9 \pm 0.5$ \\
\hline PMM-72h & $139 \pm 4$ & 15 & $15880 \pm 2150$ & $8.8 \pm 0.5$ \\
\hline BMM-30\% & $187 \pm 9$ & 30 & $16840 \pm 489$ & $11.9 \pm 0.8$ \\
\hline MMM- 0.5 wt $\%$ & $67 \pm 10^{b}$ & $0.5^{c}$ & $9778 \pm 530$ & $14.4 \pm 0.7$ \\
\hline
\end{tabular}

${ }^{a}$ Data for a MMM previously reported based on high crosslink density network-PIM-1 in PIM-1 is also shown; ${ }^{34}$ All membranes were methanoltreated and the permeation results are the average of at least 3 coupons of the same membrane type. Total feed pressure was 3 bar, and the measurements were carried out at $25^{\circ} \mathrm{C}$. The gas permeation data for fresh films were obtained $48-72 \mathrm{~h}$ after film formation (usually $24 \mathrm{~h}$ after methanol treatment). ${ }^{b}$ Data are related to the performance of PIM-1-based MMM containing 0.5 wt \% highly cross-linked network PIM-1, as reported in our previous work. ${ }^{34} c_{\text {This }}$ represents the weight percentage of the tightly cross-linked network-PIM-1 in the total PIM-1-based film.

were accurately weighed to $0.001 \mathrm{mg}$ and then placed in the analyzer furnace at $900{ }^{\circ} \mathrm{C}$. Gaseous products were separated and measured using a thermal conductivity detector.

2.5. Gas Permeation Measurements. Mixed gas permeability measurements were carried out using a binary feed mixture $(25 \mathrm{~mL}$ $\mathrm{min}^{-1}$ of each gas) in a permeation apparatus based on the standard variable volume method, as reported previously. ${ }^{34}$ The feed side pressure was kept constant at approximately 3 bar and $25{ }^{\circ} \mathrm{C}$ and the permeate side was at atmospheric pressure. Pieces of prepared freestanding membranes were masked between two aluminum-tape donuts, and the membrane-aluminum interface was sealed using twopart potting epoxy (Araldite Rapid, Industrial MTCE Suppliers). Helium $\left(60 \mathrm{~mL} \mathrm{~min}^{-1}\right)$ at atmospheric pressure was used as a sweep gas to carry the permeate to a Micro Gas Chromatograph (GC, Agilent technologies 490) for automated online analysis. The permeability of the membranes was calculated using eq 1 .

$$
P_{i}=\frac{N_{i} l}{\left(p_{i, 1}-p_{i, 2}\right)}
$$

where $P_{i}$ is the permeability coefficient for component $i$ in barrer ( 1 barrer $=10^{-10} \mathrm{~cm}^{3}[\mathrm{STP}] \mathrm{cm} \mathrm{cm}^{-2} \mathrm{~s}^{-1} \mathrm{cmHg}^{-1}=3.35 \times 10^{-16} \mathrm{~mol} \mathrm{~m}$ $\left.\mathrm{m}^{-2} \mathrm{~s}^{-1} \mathrm{~Pa}^{-1}\right), N_{i}$ is the steady state flux of penetrant $i\left(\mathrm{~cm}^{3} \mathrm{~cm}^{-2} \mathrm{~s}^{-1}\right)$, $l$ is the membrane thickness $(\mathrm{cm})$, and $p_{i, 1}$ and $p_{i, 2}$ are the partial pressures of component $i$ in the feed (1) and permeate (2) side (in $\mathrm{cmHg}$ ). The selectivity was calculated as the ratio of the permeabilities for a pair of gases. 


\section{RESULTS AND DISCUSSION}

3.1. Morphology and Density of LCD-Network-PIM-1. LCD-network-PIM-1 was found to be insoluble in organic solvents, as expected for a network structure. AFM images of LCD-network-PIM-1 deposited from a very dilute dispersion in chloroform are presented in Figure 4(a,b). Many small structures are visible, along with some ribbon-like features that may arise from agglomeration of smaller species. This is in marked contrast to the high cross-link density network-PIM-1 previously reported, ${ }^{34}$ which showed nanosheets with thicknesses in the range $3-5 \mathrm{~nm}$ and lateral dimensions of a few hundred nanometers. For the LCD-network-PIM-1, height profiles are shown in Figure 4(c) along lines passing through some of the small particles, indicated as lines $1-4$ in Figure 4(b). Heights of approximately 5, 10, and $15 \mathrm{~nm}$ can be seen. Analysis of the distribution of heights (Figure 4(d)) and the distribution of lateral dimensions (Figure 4(e)) for 100 particles from Figure 4(a) gives a number-average height of $9.7 \pm 2.8 \mathrm{~nm}$ and a number-average lateral size of $50.2 \pm 22.7$ $\mathrm{nm}$. The aspect ratio of 5:1 suggests structures that may be described as oblate spheroids. Similar structures may arise when PIM-1 is deposited from very dilute solution (see Figure $\mathrm{S} 1$ ), which in that case is most likely due to aggregation of macromolecular chains as the solvent dries. Membranes containing LCD-network-PIM-1, whether cast as a blend with preformed PIM-1 or from a polymerized mixture, were found to have significantly higher thicknesses (Table 1) than membranes formed from pure PIM-1 solutions with similar solids content. This suggests that the LCD-network-PIM-1 particles are of much lower density than a conventional PIM-1 film. Membranes containing 15 wt \% of the LCD-networkPIM-1 were around two and a half times thicker than an equivalent PIM-1 film. Given a film density of $1.09 \mathrm{~g} \mathrm{~cm}^{-3}$ for PIM-1 itself, ${ }^{37}$ this indicates a density of $0.13 \mathrm{~g} \mathrm{~cm}^{-3}$ for LCDnetwork-PIM-1. Assuming oblate spheroids with the dimensions given above, this implies a molar mass in the region of 1 $\times 10^{6} \mathrm{~g} \mathrm{~mol}^{-1}$ for the small particles of LCD-network-PIM-1 observed by AFM. We can speculate that the low density of the LCD-network-PIM-1 arises from a structure of interconnected, randomly oriented, sheet-like fragments, since we know that the high cross-link density network-PIM-1 forms nanosheets.

3.2. Effect of Grafting on LCD-Network-PIM-1. As mentioned in the Experimental Section, elemental analysis of the LCD-network-PIM-1 suggests it has some unreacted fluoro-end groups. When LCD-network-PIM-1 is included in a PIM-1 polymerization, reaction may occur at these sites, leading to grafted PIM-1 chains. Over an extended reaction time, there is the possibility of chains forming links between different network particles and covalently tying them together. In the preparation of PMM-24h and PMM-72h, where there was just $15 \mathrm{wt} \%$ LCD-network-PIM-1 in the initial polymerization mixture, it is expected that most of the PIM1 monomers will form conventional PIM-1 macromolecules, and only a small proportion will end up in chains grafted to the network.

DLS provides information about the effective sizes of species dissolved or dispersed in a medium. Number distributions of hydrodynamic diameter from DLS analysis are shown in Figure 5 for dilute dispersions in chloroform. For LCD-network-PIM1 (Figure 5(a)), apparent sizes in the range $20-100 \mathrm{~nm}$ are obtained. This is larger than might be expected on the basis of AFM analysis (see above), which may reflect some aggregation

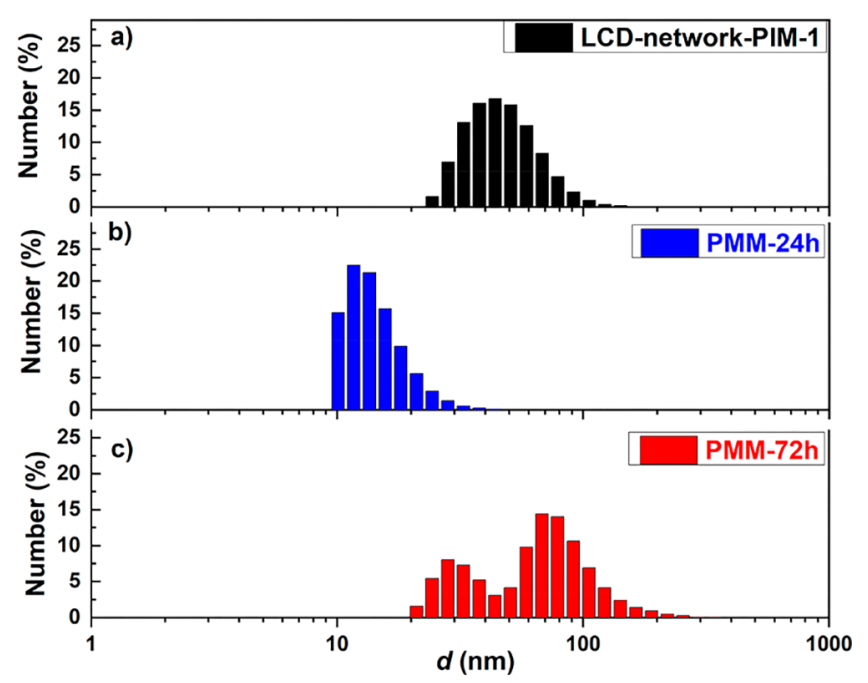

Figure 5. Number distributions of hydrodynamic diameter obtained from DLS analysis of dilute dispersions of (a) LCD-network-PIM-1, (b) PMM-24h, and (c) PMM-72h in chloroform.

in the dispersion as well as the fact that DLS analysis has some limitations for the size measurement of nonspherical particles. For PMM-24h (Figure 5(b)), the effective size is reduced to a range of $10-30 \mathrm{~nm}$, which may be interpreted as improved dispersibility arising from grafted chains. It should be noted that a pure PIM-1 solution would give a peak in the size range 1-3 nm; however, when a much larger species is also present it dominates the light scattering behavior and a smaller species, even when present in large amount, may be missed in the analysis. For PMM-72h (Figure 5(c)), which was subjected to a much longer polymerization, the apparent particle size distribution is bimodal and extends to larger sizes (up to 200 $\mathrm{nm}$ ). This may be interpreted in terms of network particles being linked together.

Multidetector SEC (see SI for full details) provides information about the soluble component of a sample. Particulate material and high molecular weight gel is filtered out prior to analysis. In the case of LCD-network-PIM-1 dispersed in chloroform, some $90 \mathrm{wt} \%$ of the sample is filtered out, consistent with it being comprised predominately of relatively large network particles. The PMM samples each contain about $15 \mathrm{wt} \%$ LCD-network-PIM-1, so that amount would be expected to be filtered out, other things being equal. In practice, it appeared that only about $10 \mathrm{wt} \%$ was filtered out in the case of PMM-24h, suggesting that some of the network was sufficiently well dispersed to pass through the filter and be included in the SEC analysis. In contrast, it appeared that about $17 \mathrm{wt} \%$ was filtered out in the case of PMM-72h, consistent with a small amount of PIM-1 being combined with the network and removed by the filter. A higher content of insoluble material in PMM-72h, as compared to PMM-24h, was also indicated by visual inspection of the respective sample solutions (see Figure S4).

The molar mass distributions determined by multidetector SEC (see SI) show that the soluble PIM-1 formed by polymerization in the presence of LCD-network-PIM-1 is similar to that which would be formed under similar conditions in the absence of network, with values of weight-average molar mass, $M_{w}$, of 100 and $143 \mathrm{~kg} \mathrm{~mol}^{-1}$ for PMM-24h and PMM$72 \mathrm{~h}$, respectively. Multidetector SEC enables intrinsic viscosity, $[\eta]$, as well as molar mass, $M$, to be determined over the range 
of molar masses in the sample. These two quantities are related by the well-known Mark-Houwink equation, $[\eta]=K M^{a}$, where $K$ and $a$ are parameters that depend on the particular polymer-solvent system. The Mark-Houwink parameter $a$ may be determined as the slope of a plot of $\log [\eta]$ against $\log M$ (see SI). Both PMM-24h and PMM-72h exhibit a slope over most of the range corresponding to a value of $a$ in the region of 0.62 , similar to conventional PIM-1, and also typical of a random coil polymer in a good solvent. However, PMM$24 \mathrm{~h}$ shows a section at molar masses in the range $350-500 \mathrm{~kg}$ $\mathrm{mol}^{-1}$ that corresponds to a relatively high $a$ value of 1.3 , which suggests the presence of a relatively rigid, anisotropic component. This represents the lower end of the molar mass range indicated by AFM analysis and density estimation, as described above. It is feasible that this represents the more dispersible portion of grafted-LCD-network-PIM-1 that passed through the filter. Additional characterization by FTIR and ${ }^{1} \mathrm{H}$ NMR spectroscopy is discussed in the SI.

3.3. $\mathrm{N}_{2}$ Sorption Behavior of LCD-Network-PIM-1 and Grafted-LCD-Network-PIM-1. Figure 6 shows $\mathrm{N}_{2}$ adsorp-

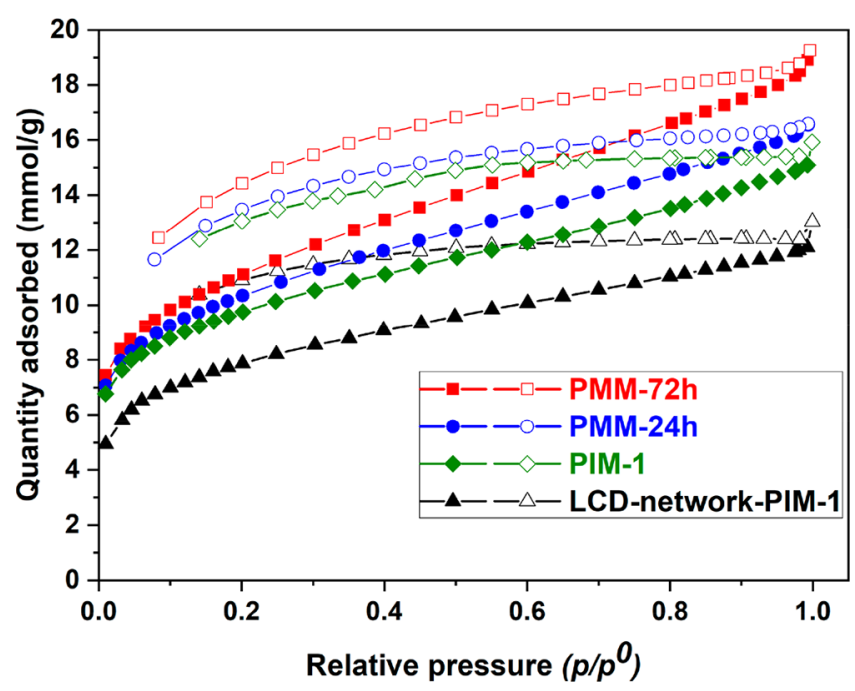

Figure 6. $\mathrm{N}_{2}$ adsorption/desorption isotherms at $77 \mathrm{~K}$ for PMM-24h and PMM-72h in comparison to LCD-network-PIM-1 and PIM-1; analysis was done on powder samples. Adsorption points are shown as filled symbols and desorption points as open symbols.

tion/desorption isotherms at $77 \mathrm{~K}$ for PIM-1, PMM-24h, PMM-72h, and LCD-network-PIM-1 powder samples. All the isotherms show high $\mathrm{N}_{2}$ adsorption at low relative pressure, as is typical of a microporous material that exhibits enhanced adsorption due to multiwall interactions. ${ }^{38}$ PMM-24h and PMM-72h, which are predominately PIM-1, show isotherms that are typical of PIM-1. The Brunauer-Emmett-Teller (BET) surface areas of PMM-24h and PMM-72h are $820 \pm 4$ and $880 \pm 4 \mathrm{~m}^{2} \mathrm{~g}^{-1}$, respectively, which is at the high end of the range of values previously reported for PIM-1. The PIM-1 sample used in this study for the preparation of pure PIM-1 membrane and blended MMMs has a BET surface area of 780 $\pm 7 \mathrm{~m}^{2} \mathrm{~g}^{-1}$. The LCD-network-PIM-1 exhibits lower $\mathrm{N}_{2}$ uptake and lower BET surface area $\left(630 \pm 3 \mathrm{~m}^{2} \mathrm{~g}^{-1}\right)$, toward the low end of the range that has been found for PIM-1, and significantly lower than the value of $940 \mathrm{~m}^{2} \mathrm{~g}^{-1}$ obtained previously for network-PIM-1 of much higher cross-link density. ${ }^{34}$ It has been found that cyclic and other nonlinear PIM-1 topologies tend to exhibit higher BET surface areas, ${ }^{13}$ so in this respect the low density LCD-network-PIM-1 behaves similarly to a linear PIM-1 sample.

3.4. Thermal Stability of LCD-Network-PIM-1 and Grafted-LCD-Network-PIM-1. Thermogravimetric analysis (TGA) curves and their first derivatives obtained from PMM24h and PMM-72h powder samples, in comparison to LCDnetwork-PIM-1 and PIM-1 powder used for the fabrication of blended MMMs, are shown in Figure 7. Small weight losses at

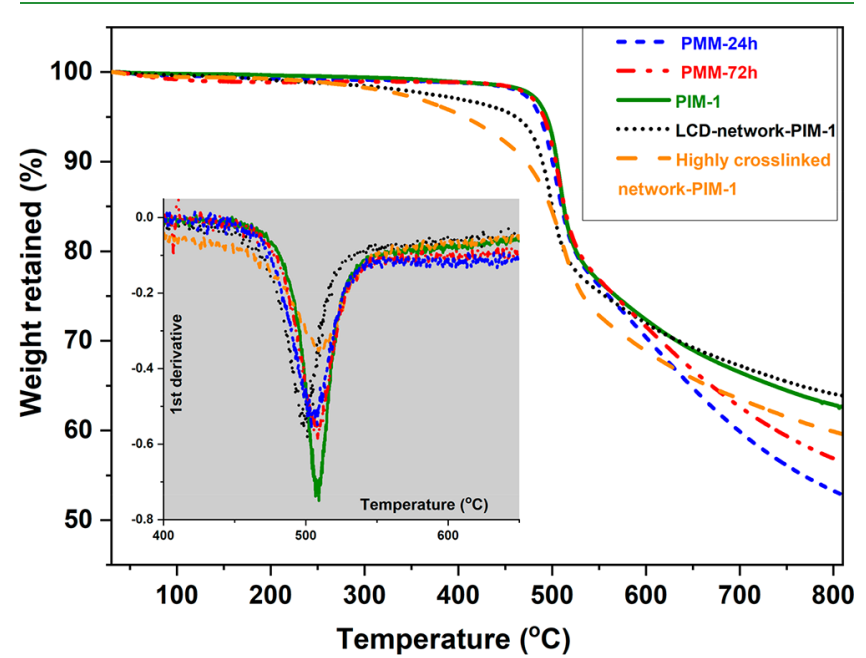

Figure 7. TGA curves and their first derivatives obtained for PMM$24 \mathrm{~h}$ and PMM-72h in comparison with LCD-network-PIM-1, PIM-1, and the previously reported highly cross-linked network-PIM-1. ${ }^{34}$

temperatures below $200{ }^{\circ} \mathrm{C}$ may be attributed to the evaporation of any volatile substances that have been trapped or adsorbed in the structure during polymerization or purification. For the PIM-1 $\left(M_{\mathrm{w}}=158 \mathrm{~kg} \mathrm{~mol}^{-1}, Ð=2.95\right)$, and for PMM-24h and PMM-72h which are predominately PIM-1, there is no significant thermal degradation of the polymeric backbone under the conditions of the TGA experiment until around $450{ }^{\circ} \mathrm{C}$. For LCD-network-PIM-1 the onset of degradation is around $300{ }^{\circ} \mathrm{C}$. This lower onset of degradation is similar to the behavior observed previously for network-PIM-1 of much higher cross-link density, ${ }^{34}$ also shown in Figure 7 , which was attributed to the presence of some labile short branches in the structure. The grafting reaction seems to have either extended or eliminated such short branches, as there is no sign of early degradation in the samples that include grafted-LCD-network-PIM-1.

3.5. Mixed Matrix Membranes and Gas Separation. PMM-24h and PMM-72h (prepared by carrying out a PIM-1 polymerization in the presence of $15 \mathrm{wt} \%$ LCD-network-PIM1 for 24 and $72 \mathrm{~h}$, respectively) were used to fabricate MMMs without any further addition of PIM-1 or filler. For comparison, MMMs were also fabricated by the conventional blending and casting route, using two loadings of LCDnetwork-PIM-1 (15 and 30 wt \% with respect to the total dry solids content, indicated as BMM-15\% and BMM-30\%, respectively). These filler loadings are higher than could be achieved in our previous work with highly cross-linked network-PIM-1, ${ }^{34}$ where the amount of filler that could be used with PIM-1 to form mechanically stable MMMs was limited to $10 \mathrm{wt} \%$, a problem that was attributed to the nanosheet morphology of the tightly cross-linked filler. The higher loadings achievable with the lower cross-link density filler indicate its better compatibility. Nevertheless, some 
inhomogeneity was observed in membranes prepared by blending, and to a lesser extent in those formed with the grafted-LCD-network-PIM-1 samples (see Figure S12).

A cross-sectional SEM image of the blended membrane BMM-15\% (Figure 1(e)) shows some potential defect sites, whereas the PMM-24h membrane (Figure 1(f)) with essentially the same network content appears much more homogeneous, consistent with enhanced compatibility arising from the grafting reaction. However, the product of the extended grafting reaction, PMM-72h, gave membranes that were less homogeneous (Figure $1(\mathrm{~g})$ ), which may be related to the larger structures formed by linking of LCD-network-PIM-1 particles as discussed above.

Table 1 summarizes initial $\mathrm{CO}_{2} / \mathrm{CH}_{4}$ gas separation data obtained for membranes formed from the products of grafting reactions, PMM-24h and PMM-72h, and for MMMs prepared by the blending and casting route, BMM-15\% and BMM-30\%, in comparison with a pure PIM-1 membrane.

The addition of LCD-network-PIM-1 to PIM-1 by either route noticeably enhances the permeability at the expense of some selectivity, reflecting the low density of the filler, as discussed above. The permeance (permeability/thickness) is actually marginally higher for the pure PIM-1 than for PMM$24 \mathrm{~h}$ or the BMM samples. When the grafted product PMM$24 \mathrm{~h}$ is compared with the blended membrane of comparable network content, BMM-15\%, the blended membrane shows slightly higher permeability and lower selectivity, which may be the result of defects as suggested by the SEM image in Figure 1(e). Table 1 also includes data for a MMM with much lower loading of tightly cross-linked network-PIM-1 as reported previously. ${ }^{34}$ In contrast to the LCD-network-PIM-1 explored in the present work, the tightly cross-linked network-PIM-1 nanosheets improve both selectivity and permeability at very low loading.

The long-term aging behavior of a membrane composed of a high free volume, glassy polymer is of particular concern when considering its potential commercial use. The $\mathrm{CO}_{2} / \mathrm{CH}_{4}$ mixed-gas separation performance of PMM-24h over 160 days is shown in Figure 8. There is some loss of permeability, from an average of 12510 barrer for fresh methanol-treated samples to almost 8900 barrer for 160-day aged samples, but

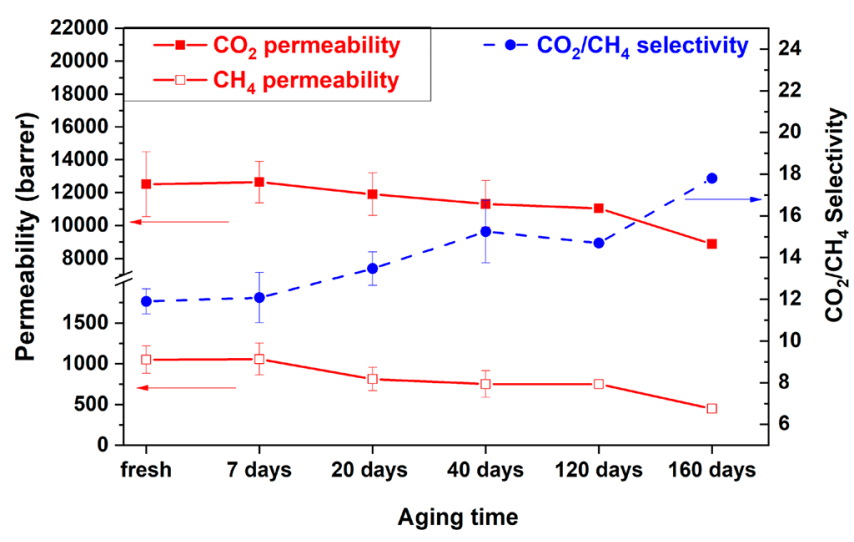

Figure 8. $\mathrm{CO}_{2} / \mathrm{CH}_{4}$ mixed gas $(1: 1, \mathrm{v} / \mathrm{v})$ separation results obtained for methanol-treated PMM-24h membranes over 160 days; the total feed pressure was $3 \mathrm{bar}$, and measurements were done at $25^{\circ} \mathrm{C}$. Error bars represent the standard deviation of values measured for 3 membrane coupons. this is accompanied by an increase in the $\mathrm{CO}_{2} / \mathrm{CH}_{4}$ selectivity from 11.9 to 17.8 .

Physical aging of high free volume PIM-1-based membranes often follows two stages. The first phase of physical aging usually includes relaxation of the excess free volume caused by methanol treatment of the films, which results in a rapid decrease in the permeability. In the second phase, it is believed that reorganization of the free volume of the film causes a more gradual decrease in permeability. ${ }^{39,40}$ Interestingly, it was observed here that the decrease in the permeability (physical aging) of the MMM that was prepared based on the grafting reaction (PMM-24h) occurred very gradually during the overall period of 160 days. This result might indicate some partial entanglement of the PIM-1 chains by the LCDnetwork-PIM-1 particles, which could help maintain the initial high free volume of the polymeric chains. Similar results have also been reported in the literature by addition of porous aromatic frameworks (PAFs) to high free volume polymers such as PIM-1 and poly(trimethylsilylpropyne) (PTMSP). ${ }^{29}$ It has been shown that microporous PAF particles can form an interwoven nanocomposite with high free volume polymers, thus holding open the pores of the polymeric material.

Figure 9 shows the mixed gas aging behavior of PMM-24h on a double logarithmic plot of $\mathrm{CO}_{2} / \mathrm{CH}_{4}$ selectivity against

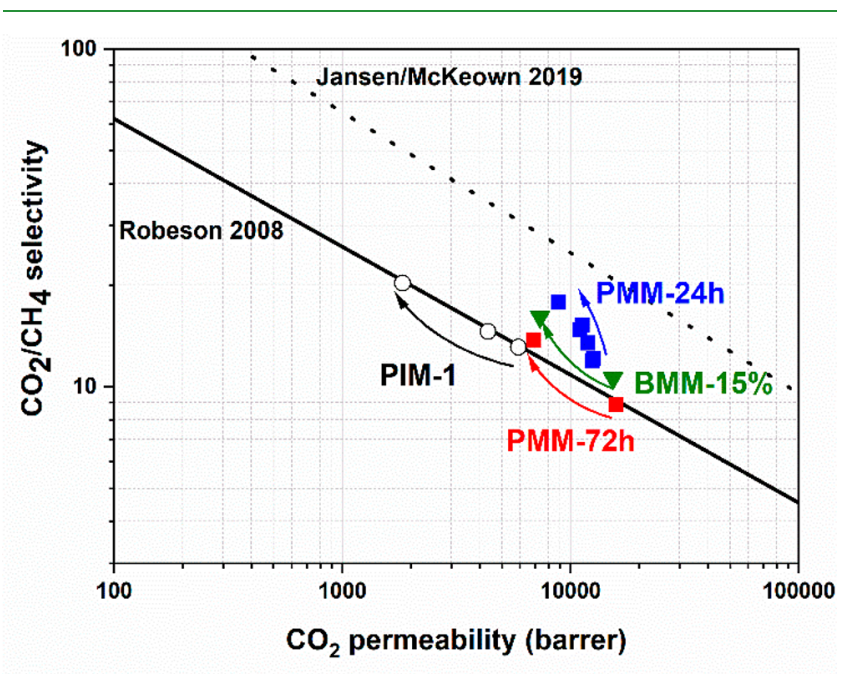

Figure 9. Robeson plot showing the aging behavior of MMMs containing 15 wt \% LCD-network-PIM-1 (PMM-24h, PMM-72h, and BMM-15\%) and PIM-1 membranes for the separation of $\mathrm{CO}_{2} / \mathrm{CH}_{4}$ $(1: 1, \mathrm{v} / \mathrm{v})$ mixtures at $25{ }^{\circ} \mathrm{C}$ and $3 \mathrm{bar}$ total feed pressure; The last data points for PMM-24h, BMM-15\%, and PIM-1 are related to 160 day aged films and for PMM-72h is related to 120-day aged film; all membranes were initially methanol treated; The solid line represents the 2008 upper bound, and the dotted line shows the recently proposed 2019 upper bound. ${ }^{12}$ The arrows point toward the most aged membranes.

$\mathrm{CO}_{2}$ permeability, and compares it with that of PMM-72h, a blended membrane (BMM-15\%) and a pure PIM-1 membrane (detailed data are presented in Table S3). All the membranes were aged for 160 days, except PMM-72h, for which the last data point is related to a 120 -day aged film. The plot includes the 2008 Robeson upper bound, ${ }^{6}$ and the recently proposed upper bound of Jansen and McKeown. ${ }^{12}$ It can be seen that while PIM-1 loses $69 \%$ of its initial $\mathrm{CO}_{2}$ permeability after 160 days, the decrease in $\mathrm{CO}_{2}$ permeability of the PMM-24h membrane is limited to only $29 \%$ over the same period of time. 
Indeed, after 160 days the $\mathrm{CO}_{2}$ permeability of PMM-24h ( 8870 barrer) remains higher than that of the fresh PIM-1 membrane (5920 barrer). Furthermore, the data points for PMM-24h lie above the 2008 upper bound and tend toward the recently proposed 2019 upper bound, whereas the data points for PIM-1 lie along the 2008 upper bound. If some aging is regarded as inevitable for high free volume, glassy polymers, then aging that tends upward in a Robeson plot is most desirable. The 120-day aged PMM-72h also seems to have followed a similar path to that of the PIM-1 membrane, with the data points related to both fresh and aged PMM-72h lying very close to the 2008 Robeson upper bound, and a loss of $56 \%$ from the initially high $\mathrm{CO}_{2}$ permeability after 120 days of aging. The blended membrane BMM-15\%, with a $48 \%$ loss in $\mathrm{CO}_{2}$ permeability, also shows improved aging behavior compared to PIM-1, although not quite as dramatically as PMM-24h. Thus, although fresh BMM-15\% has a higher permeability than PMM-24h, after 160 days it has dropped to a lower value than PMM-24h. These results clearly demonstrate the significant effect of the addition of LCD-network-PIM-1, and the additional value of grafting reactions, as tools to control aging of PIM-1-based membranes.

Figure 10 compares the aging behavior of PMM-24h and $\mathrm{BMM}-15 \%$ from this work with data from the literature for some other membranes based on high free volume glassy

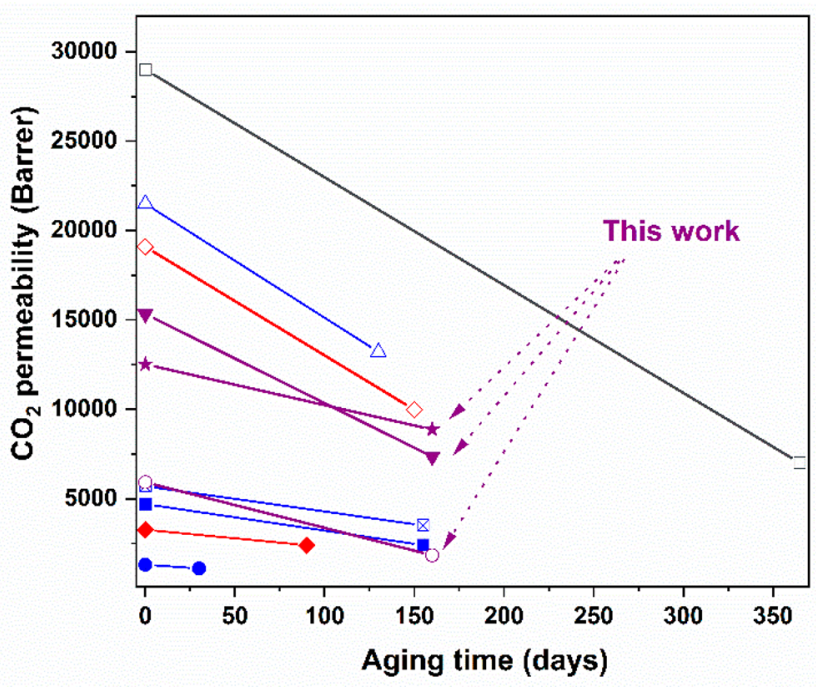

Figure 10. Changes in $\mathrm{CO}_{2}$ permeabilities over time for PIM-1 and two MMMs prepared in this work (PMM-24h, BMM-15\%) in comparison to some other high free volume membranes based on PIMs and PTMSP in the literature for $\mathrm{CO}_{2}$ separation; these membranes include: $\square$, PTMSP $(76 \%$, film thickness $40-70 \mu \mathrm{m}){ }^{39}$ blue $\square$, PIM-1/0.05rGO-octadecylamine (ODA) (49\%, film thickness $\sim 50 \mu \mathrm{m})$; ${ }^{25}$ blue $\square$ with an $\times$ in the center, PIM-1/0.05 rGOoctylamine(OA) (39\%, film thickness $\sim 50 \mu \mathrm{m}){ }^{25}$ blue $\bullet$, PIM-1/10 wt \% PEG-polyhedral oligomeric silsesquioxane(POSS) (16\%, film thickness $70-100 \mu \mathrm{m}){ }^{41}$ red $\diamond$, PIM- $1 / 5$ wt $\%$ amino-functionalized POSS $(27 \%$, film thickness $70-80 \mu \mathrm{m}){ }^{42}$ red $\diamond$, PIM-1/16.7 wt $\%$ hyper-cross-linked polystyrene (HCP, film thickness $\sim 120 \mu \mathrm{m}$ ) $(47 \%){ }^{26}$ blue $\triangle$, PIM-BTrip (39\%, film thickness $\left.160 \mu \mathrm{m}\right) ;^{12}$ purple $\boldsymbol{\nabla}$, BMM- $15 \%$ this work (48\%, film thickness $150 \mu \mathrm{m})$; purple $\star$, PMM-24h this work (29\%, film thickness $140 \mu \mathrm{m})$; purple $\bigcirc$, PIM-1 this work $(69 \%$, film thickness $60 \mu \mathrm{m})$; the numbers in parentheses show the $\%$ reduction in the initial $\mathrm{CO}_{2}$ permeability of the membranes over the aging time; and the lines in the graph are for the purpose of guiding the eye. polymers. It can be seen that while higher initial permeabilities have been obtained for some other systems, PMM-24h is significantly better at retaining its permeability over 160 days. This highlights the importance of studying membrane behavior over time, and not just reporting initial performance.

\section{CONCLUSIONS}

Recently, there has been a drive to explore the problem of physical aging for high free volume, glassy polymers, such as PIM-1, in order to realize the full potential of these materials for large-scale membrane-based separations. One way to control aging is through the addition of fillers to give MMMs, but many fillers show poor compatibility with a polymeric continuous phase. Here we have demonstrated an effective filler based on the same structural motif as the continuous phase, creating a network version of PIM-1 dispersed in PIM-1 itself.

Our previous work on high cross-link density network-PIM1 gave rise to a nanosheet morphology that offers enhanced $\mathrm{CO}_{2} / \mathrm{CH}_{4}$ separation performance, but that can only be dispersed at low loading in PIM-1. Here we have shown that low cross-link density LCD-network-PIM-1, which forms spheroidal rather than sheet structures, can be dispersed at higher loadings. We have also shown that compatibility can be further improved by a grafting reaction, achieved by including LCD-network-PIM-1, which has residual fluoro- groups, in a PIM-1 polymerization. However, if the grafting reaction is continued for too long a time ( $72 \mathrm{~h}$ rather than $24 \mathrm{~h}$ ), then the network particles are themselves linked together, increasing the particle size and introducing defects that lead to a deterioration in membrane performance.

For membranes with grafted-LCD-network-PIM-1 after $24 \mathrm{~h}$ reaction, PMM-24h, a good combination of initial $\mathrm{CO}_{2}$ permeability (12,510 barrer) and $\mathrm{CO}_{2} / \mathrm{CH}_{4}$ selectivity (11.9) was obtained. PMM-24h showed well-controlled aging behavior over 160 days, with only $29 \%$ reduction of the initial $\mathrm{CO}_{2}$ permeability, as compared to $69 \%$ reduction for a pure PIM-1 membrane. Furthermore, PMM-24h not only surpassed the 2008 upper bound, but tended toward the 2019 upper limit. In comparison, a conventional MMM of similar composition (BMM-15\%), formed by blending and casting LCD-network-PIM-1 with PIM-1, while having a higher initial $\mathrm{CO}_{2}$ permeability (14470 barrer), exhibited a $48 \%$ reduction over the same period.

These results demonstrate the great potential of network formation and grafting for the development of membranes with long-term stable gas separation performance.

\section{ASSOCIATED CONTENT}

SI Supporting Information

The Supporting Information is available free of charge at https://pubs.acs.org/doi/10.1021/acsami.0c13838.

Experimental details for syntheses of PIM-1 and DCOB monomer, characterization results for LCD-networkPIM-1 and the grafted samples, as well as gas separation data of fresh and aged membranes (PDF)

\section{AUTHOR INFORMATION}

\section{Corresponding Author}

Peter M. Budd - Department of Chemistry, University of Manchester, M13 9PL Manchester, United Kingdom; 
다이.org/0000-0003-3606-1158; Email: Peter.Budd@

manchester.ac.uk

\section{Authors}

Marzieh Tamaddondar - Department of Chemistry, University of Manchester, M13 9PL Manchester, United Kingdom

Andrew B. Foster - Department of Chemistry, University of Manchester, M13 9PL Manchester, United Kingdom; (1) orcid.org/0000-0001-8222-673X

Mariolino Carta - Department of Chemistry, College of Science, Swansea University, SA2 8PP Swansea, United Kingdom; (1) orcid.org/0000-0003-0718-6971

Patricia Gorgojo - Department of Chemical Engineering and Analytical Science, University of Manchester, M13 9PL Manchester, United Kingdom; 이이이.org/0000-0002-6905$714 \mathrm{X}$

Neil B. McKeown - EastChem, School of Chemistry, University of Edinburgh, EH9 3FJ Edinburgh, United Kingdom; (1) orcid.org/0000-0002-6027-261X

Complete contact information is available at: https://pubs.acs.org/10.1021/acsami.0c13838

\section{Author Contributions}

M.T. designed and carried out the experimental work and prepared the manuscript under the direction of P.G. and P.M.B. A.B.F. analyzed the NMR, SEC, and elemental analysis results of the grafted products and also wrote the relevant sections. M.C. and N.B.M. provided guidance on monomer synthesis. All authors have contributed to the revision of the manuscript and have given approval to the final version.

\section{Funding}

The authors gratefully acknowledge funding from the Engineering and Physical Sciences Research Council (EPSRC) Programme Grant EP/M01486X/1 "From membrane material synthesis to fabrication and function (SynFabFun)" and the University of Manchester.

\section{Notes}

The authors declare no competing financial interest.

\section{ABBREVIATIONS USED}

PIM, polymer of intrinsic microporosity

LCD, low cross-link density

DCOB, dicyanooctafluoro biphenyl

TFTPN, tetrafluoroterephthalonitrile

TTSBI, 5,5',6,6' '-tetrahydroxy-3,3,3',3' -tetramethyl-1,1' -spirobisindane

MMMs, mixed matrix membranes

PMM, polymerized mixed matrix

BMM, blended mixed matrix

BET, Brunauer-Emmett-Teller

SEC, size exclusion chromatography

NMR, nuclear magnetic resonance

FTIR, Fourier transform infrared

ATR, attenuated total reflectance

AFM, atomic force microscopy

TGA, thermogravimetric analysis

\section{REFERENCES}

(1) Sanders, D. F.; Smith, Z. P.; Guo, R.; Robeson, L. M.; McGrath, J. E.; Paul, D. R.; Freeman, B. D. Energy-Efficient Polymeric Gas Separation Membranes for a Sustainable Future: A Review. Polymer 2013, 54, 4729-4761.
(2) Park, H. B.; Kamcev, J.; Robeson, L. M.; Elimelech, M.; Freeman, B. D. Maximizing the Right Stuff: The Trade-off Between Membrane Permeability and Selectivity. Science 2017, 356, No. eaab0530.

(3) Budd, P. M.; Msayib, K. J.; Tattershall, C. E.; Ghanem, B. S.; Reynolds, K. J.; McKeown, N. B.; Fritsch, D. Gas Separation Membranes from Polymers of Intrinsic Microporosity. J. Membr. Sci. 2005, 251, 263-269.

(4) Sing, K. S. W. Reporting Physisorption Data for Gas/Solid Systems with Special Reference to the Determination of Surface Area and Porosity (Recommendations 1984). Pure Appl. Chem. 1985, 57, 603-619.

(5) Robeson, L. M. Correlation of Separation Factor versus Permeability for Polymeric Membranes. J. Membr. Sci. 1991, 62, $165-185$.

(6) Robeson, L. M. The Upper Bound Revisited. J. Membr. Sci. 2008, 320, 390-400.

(7) Freeman, B. D. Basis of Permeability/Selectivity Tradeoff Relations in Polymeric Gas Separation Membranes. Macromolecules 1999, 32, 375-380.

(8) Carta, M.; Croad, M.; Malpass-Evans, R.; Jansen, J. C.; Bernardo, P.; Clarizia, G.; Friess, K.; Lanč, M.; McKeown, N. B. Triptycene Induced Enhancement of Membrane Gas Selectivity for Microporous Tröger's Base Polymers. Adv. Mater. 2014, 26, 3526-3531.

(9) Carta, M.; Malpass-Evans, R.; Croad, M.; Rogan, Y.; Jansen, J. C.; Bernardo, P.; Bazzarelli, F.; McKeown, N. B. An Efficient Polymer Molecular Sieve for Membrane Gas Separations. Science 2013, 339, 303-307.

(10) Rose, I.; Bezzu, C. G.; Carta, M.; Comesaña-Gandara, B.; Lasseuguette, E.; Ferrari, M. C.; Bernardo, P.; Clarizia, G.; Fuoco, A.; Jansen, J. C.; Hart, K. E.; Liyana-Arachchi, T. P.; Colina, C. M.; McKeown, N. B. Polymer Ultrapermeability from the Inefficient Packing of 2D Chains. Nat. Mater. 2017, 16, 932-937.

(11) Swaidan, R.; Ghanem, B.; Pinnau, I. Fine-tuned Intrinsically Ultramicroporous Polymers Redefine the Permeability/Selectivity Upper Bounds of Membrane-based Air and Hydrogen Separations. ACS Macro Lett. 2015, 4, 947-951.

(12) Comesaña-Gándara, B.; Chen, J.; Bezzu, C. G.; Carta, M.; Rose, I.; Ferrari, M.-C.; Esposito, E.; Fuoco, A.; Jansen, J. C.; McKeown, N. B. Redefining the Robeson Upper Bounds for $\mathrm{CO}_{2} / \mathrm{CH}_{4}$ and $\mathrm{CO}_{2} / \mathrm{N}_{2}$ Separations Using a Series of Ultrapermeable Benzotriptycene-based Polymers of Intrinsic Microporosity. Energy Environ. Sci. 2019, 12, 2733-2740.

(13) Foster, A. B.; Tamaddondar, M.; Luque-Alled, J. M.; Harrison, W. J.; Li, Z.; Gorgojo, P.; Budd, P. M. Understanding the Topology of the Polymer of Intrinsic Microporosity PIM-1: Cyclics, Tadpoles and Network Structures and their Impact on Membrane Performance. Macromolecules 2020, 53, 569-583.

(14) Low, Z.-X.; Budd, P. M.; McKeown, N. B.; Patterson, D. A. Gas Permeation Properties, Physical Aging, and its Mitigation in High Free Volume Glassy Polymers. Chem. Rev. 2018, 118, 5871-5911.

(15) Du, N.; Cin, M. M. D.; Pinnau, I.; Nicalek, A.; Robertson, G. P.; Guiver, M. D. Azide-based Cross-Linking of Polymers of Intrinsic Microporosity (PIMs) for Condensable Gas Separation. Macromol. Rapid Commun. 2011, 32, 631-636.

(16) Du, N.; Dal-Cin, M. M.; Robertson, G. P.; Guiver, M. D. Decarboxylation-induced Cross-linking of Polymers of Intrinsic Microporosity (PIMs) for Membrane Gas Separation. Macromolecules 2012, 45, 5134-5139.

(17) Song, Q.; Cao, S.; Pritchard, R. H.; Ghalei, B.; Al-Muhtaseb, S. A.; Terentjev, E. M.; Cheetham, A. K.; Sivaniah, E. Controlled Thermal Oxidative Crosslinking of Polymers of Intrinsic Microporosity Towards Tunable Molecular Sieve Membranes. Nat. Commun. 2014, 5, 4813-4825.

(18) Li, F. Y.; Xiao, Y.; Chung, T.-S.; Kawi, S. High-Performance Thermally Self-Cross-Linked Polymer of Intrinsic Microporosity (PIM-1) Membranes for Energy Development. Macromolecules 2012, 45, 1427-1437. 
(19) Khan, M. M.; Bengtson, G.; Shishatskiy, S.; Gacal, B. N.; Mushfequr Rahman, M.; Neumann, S.; Filiz, V.; Abetz, V. Crosslinking of Polymer of Intrinsic Microporosity (PIM-1) via Nitrene Reaction and its Effect on Gas Transport Property. Eur. Polym. J. 2013, 49, 4157-4166.

(20) Khan, M. M.; Filiz, V.; Bengtson, G.; Rahman, M. M.; Shishatskiy, S.; Abetz, V. Functionalized Carbon Nanotube Mixed Matrix Membranes of Polymers of Intrinsic Microporosity (PIMs) for Gas Separation. Procedia Eng. 2012, 44, 1899-1901.

(21) Song, Q.; Cao, S.; Pritchard, R. H.; Qiblawey, H.; Terentjev, E. M.; Cheetham, A. K.; Sivaniah, E. Nanofiller-tuned Microporous Polymer Molecular Sieves for Energy and Environmental Processes. J. Mater. Chem. A 2016, 4, 270-279.

(22) Wang, Z.; Ren, H.; Zhang, S.; Zhang, F.; Jin, J. Polymers of Intrinsic Microporosity/Metal-Organic Framework Hybrid Membranes with Improved Interfacial Interaction for High-performance $\mathrm{CO}_{2}$ Separation. J. Mater. Chem. A 2017, 5, 10968-10977.

(23) Jiang, H.; Zhang, J.; Huang, T.; Xue, J.; Ren, Y.; Guo, Z.; Wang, H.; Yang, L.; Yin, Y.; Jiang, Z.; Guiver, M. D. Mixed-Matrix Membranes with Covalent Triazine Framework Fillers in Polymers of Intrinsic Microporosity for $\mathrm{CO}_{2}$ Separations. Ind. Eng. Chem. Res. 2020, 59, 5296-5306.

(24) Wu, X.; Ren, Y.; Sui, G.; Wang, G.; Xu, G.; Yang, L.; Wu, Y.; He, G.; Nasir, N.; Wu, H.; Jiang, Z. Accelerating $\mathrm{CO}_{2}$ Capture of Highly Permeable Polymer through Incorporating Highly Selective Hollow Zeolite Imidazolate Framework. AIChE J. 2020, 66, No. e16800.

(25) Alberto, M.; Bhavsar, R.; Luque-Alled, J. M.; Vijayaraghavan, A.; Budd, P. M.; Gorgojo, P. Impeded Physical Aging in PIM-1 Membranes Containing Graphene-like Fillers. J. Membr. Sci. 2018, $563,513-520$.

(26) Mitra, T.; Bhavsar, R. S.; Adams, D. J.; Budd, P. M.; Cooper, A. I. PIM-1 Mixed Matrix Membranes for Gas Separations using Costeffective Hypercrosslinked Nanoparticle Fillers. Chem. Commun. 2016, 52, 5581-5584.

(27) Bhavsar, R. S.; Mitra, T.; Adams, D. J.; Cooper, A. I.; Budd, P. M. Ultrahigh-permeance PIM-1 Based Thin Film Nanocomposite Membranes on PAN Supports for $\mathrm{CO}_{2}$ Separation. J. Membr. Sci. 2018, 564, 878-886.

(28) Lau, C. H.; Mulet, X.; Konstas, K.; Doherty, C. M.; Sani, M.-A.; Separovic, F.; Hill, M. R.; Wood, C. D. Hypercrosslinked Additives for Ageless Gas-Separation Membranes. Angew. Chem., Int. Ed. 2016, 55, 1998-2001.

(29) Lau, C. H.; Nguyen, P. T.; Hill, M. R.; Thornton, A. W.; Konstas, K. C.; Doherty, M.; Mulder, R. J.; Bourgeois, L.; Liu, A. C. Y.; Sprouster, D. J.; Sullivan, J. P.; Bastow, T. J.; Hill, A. J.; Gin, D. L.; Noble, R. D. Ending Aging in Super Glassy Polymer Membranes. Angew. Chem., Int. Ed. 2014, 53, 5322-5326.

(30) Hou, R.; Smith, S. J. D.; Wood, C. D.; Mulder, R. J.; Lau, C. H.; Wang, H.; Hill, M. R. Solvation Effects on the Permeation and Aging Performance of PIM-1-based MMMs for Gas Separation. ACS Appl. Mater. Interfaces 2019, 11, 6502-6511.

(31) Jiang, Y.; Liu, C.; Caro, J.; Huang, A. A New UiO-66- $\mathrm{NH}_{2}$ Based Mixed-Matrix Membranes with High $\mathrm{CO}_{2} / \mathrm{CH}_{4}$ Separation Performance. Microporous Mesoporous Mater. 2019, 274, 203-211.

(32) Friebe, S.; Mundstock, A.; Volgmann, K.; Caro, J. On the Better Understanding of the Surprisingly High Performance of MetalOrganic Framework-Based Mixed-Matrix Membranes Using the Example of UiO-66 and Matrimid. ACS Appl. Mater. Interfaces 2017, 9, 41553-41558.

(33) Tien-Binh, N.; Rodrigue, D.; Kaliaguine, S. In-situ Cross Interface Linking of PIM-1 Polymer and UiO-66- $\mathrm{NH}_{2}$ for Outstanding Gas Separation and Physical Aging Control. J. Membr. Sci. 2018, 548, 429-438.

(34) Tamaddondar, M.; Foster, A. B.; Luque-Alled, J. M.; Msayib, K. J.; Carta, M.; Sorribas, S.; Gorgojo, P.; McKeown, N. B.; Budd, P. M. Intrinsically Microporous Polymer Nanosheets for High-performance Gas Separation Membranes. Macromol. Rapid Commun. 2020, 41, 1900572.
(35) Althumayri, K.; Harrison, W. J.; Shin, Y.; Gardiner, J. M.; Casiraghi, C.; Budd, P. M.; Bernardo, P.; Clarizia, G.; Jansen, J. C. The Influence of Few-layer Graphene on the Gas Permeability of the High-free-volume Polymer PIM-1. Philos. Trans. R. Soc., A 2016, 374, 20150031.

(36) Tian, Z.; Wang, S.; Wang, Y.; Ma, X.; Cao, K.; Peng, D.; Wu, X.; Wu, H.; Jiang, Z. Enhanced Gas Separation Performance of Mixed Matrix Membranes from Graphitic Carbon Nitride Nanosheets and Polymers of Intrinsic Microporosity. J. Membr. Sci. 2016, 514, 15-24.

(37) Budd, P. M.; McKeown, N. B.; Fritsch, D. Polymers of Intrinsic Microporosity (PIMs): High Free Volume Polymers for Membrane Applications. Macromol. Symp. 2006, 245-246, 403-405.

(38) Dubinin, M. M. Fundamentals of the Theory of Adsorption in Micropores of Carbon Adsorbents: Characteristics of their Adsorption Properties and Microporous Structures. Carbon 1989, 27, 457-467.

(39) Lau, C. H.; Konstas, K.; Doherty, C. M.; Kanehashi, S.; Ozcelik, B.; Kentish, S. E.; Hill, A. J.; Hill, M. R. Tailoring Physical Aging in Super Glassy Polymers with Functionalized Porous Aromatic Frameworks for $\mathrm{CO}_{2}$ Capture. Chem. Mater. 2015, 27, 4756-4762.

(40) Galizia, M.; Chi, W. S.; Smith, Z. P.; Merkel, T. C.; Baker, R. W.; Freeman, B. D. 50th Anniversary Perspective: Polymers and Mixed Matrix Membranes for Gas and Vapor Separation: A Review and Prospective Opportunities. Macromolecules 2017, 50, 7809-7843.

(41) Yang, L.; Tian, Z.; Zhang, X.; Wu, X.; Wu, Y.; Wang, Y.; Peng, D.; Wang, S.; Wu, H.; Jiang, Z. Enhanced $\mathrm{CO}_{2}$ Selectivities by Incorporating $\mathrm{CO}_{2}$-philic PEG-POSS into Polymers of Intrinsic Microporosity Membrane. J. Membr. Sci. 2017, 543, 69-78.

(42) Kinoshita, Y.; Wakimoto, K.; Gibbons, A. H.; Isfahani, A. P.; Kusuda, H.; Sivaniah, E.; Ghalei, B. Enhanced PIM-1 Membrane Gas Separation Selectivity through Efficient Dispersion of Functionalized POSS Fillers. J. Membr. Sci. 2017, 539, 178-186. 